in the desiccator under the drying condition, the total activity was 30.8 units. Then, the desmoenzyme activity was preserved for 40 per cent (14.8 units) of the total, while the lyoenzyme counted 11.7 units (32\%). In case of pre-fixation of the tissue with acetone, the total phosphorylase was considerably decreased and showed 28.1 units. The desmoenzyme activity was preserved only for about 16 per cent. Alcohol remarkably disturbed the enzyme activity and the desmoenzyme was kept only for 14 per cent in the tissue. Formalin destroyed completely the enzyme activity (see figure).

From this experiment, it was considered that for the histochemical demonstration of tissue phosphorylase the cryostat cut section should be first completely dried on obiect glass. In practice, it was found that the richest activity could be histochemically preserved in the tissue sections, when they were sufficiently dried at $7^{\circ} \mathrm{C}$ for one hour and were kept at $-20^{\circ} \mathrm{C}$ in the deep freezer for 24 hours under the drying condition.

\title{
Influence of Glutaraldehyde Fixation on Acid
}

$$
\text { Phosphatase Reaction }
$$

\author{
Masanori Akasaka. \\ Department of Pathology, Kumamoto University School of Medicine, Kumamoto
}

It is not completly known up to the persent whether the electron micrograph using the lead nitrate technnique for acid phosphatase reveals a specific reaction and shows whole intracellular distribution. Glutaraldehyde is known to be suitable for fixation of the enzyme activity in electron microscopic research, but a few problems which disturb it remain yet. Influence of glutaraldehyde and other fixatives on acid phosphatase reaction was observed in this paper, particularly in the histochemical level.

\section{Materials and Methods}

1) The smear materials of $\mathrm{AH}-13$ ascites hepatoma cells of rat were fixed in various times respectivey. They were then incubated in the Gomori modified substrate mixture for various time respectively, were observed and compared each other the lead sulfide technique.

2) The coagulated materials containing cancer cells picked up from the rat ascites were fixed in $6.3 \%$ glutaraldehyde and incubated for various times respectively. Following the materials were dehydrated with alcohol and were embedded in paraffin, they were observed by the above mentioned histochemical technique. On the other hand, after the materials were refixed in osmium, 


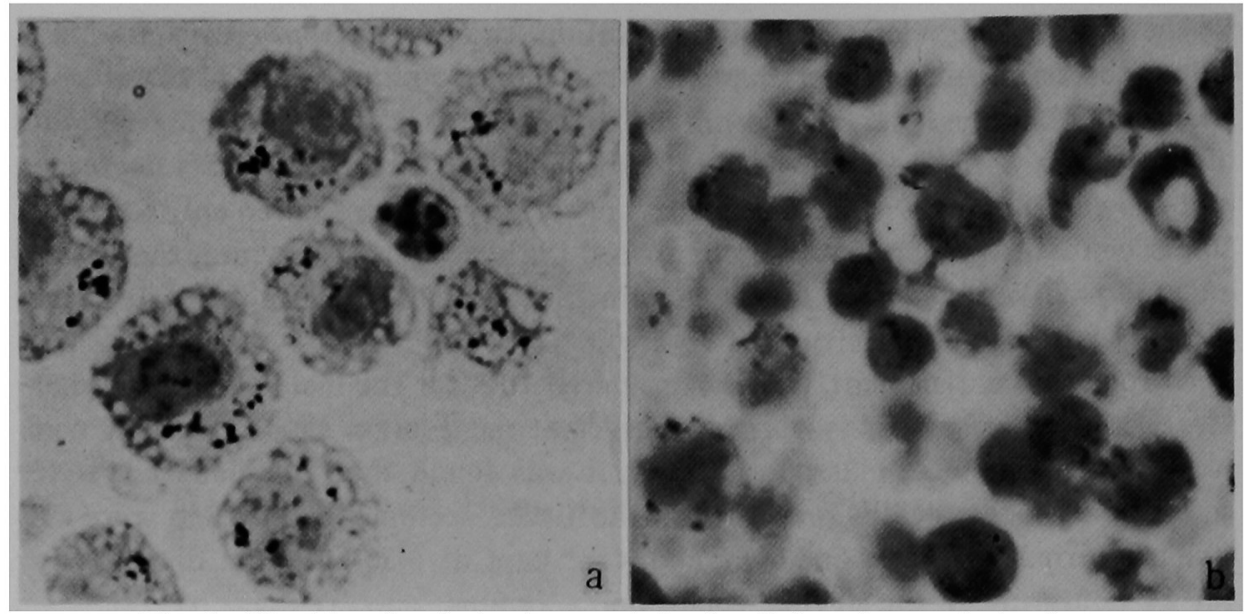

Fig. 1 The histochemical micrograph in the light microscopic level are shown. The final products for the acid phosphatase reaction appeared in granular shape in the particular regions of cytoplasm on the smear material (a) as well as in the coagulated material (b).

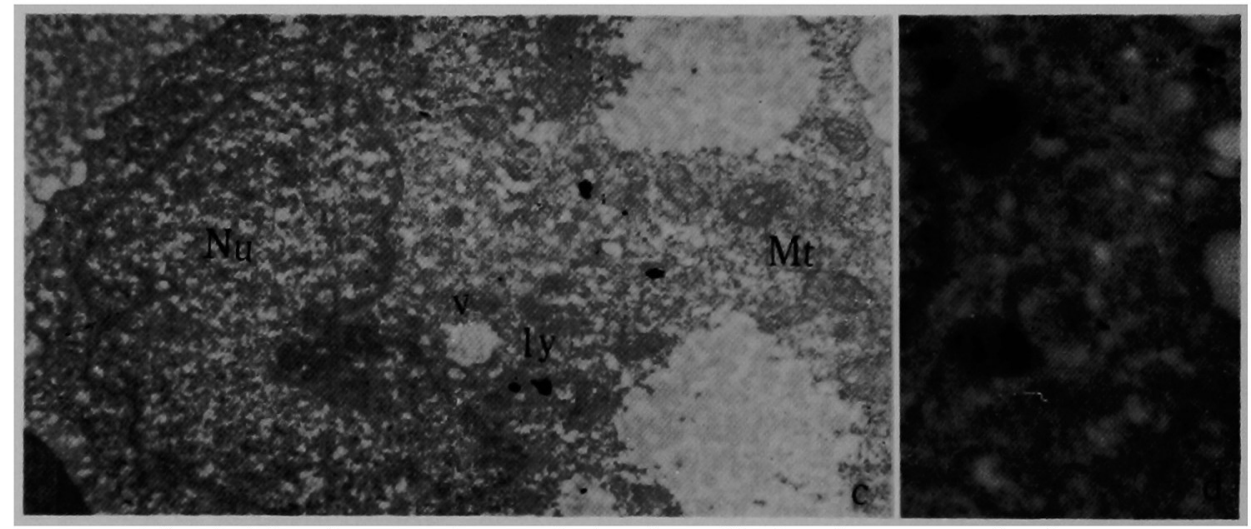

Fig. 2 The electron micrograph for the acid phosphatase reaction is revealed, demonstrating the enzyme activity in lysosomes (c). The lead precipitation for the acid phosphatase occurred in lysosomes (d).

dehydrated with alcohol and embedded in Epon, they were observed by the electron microscopic technique.

\section{Result}

1) The histochemical reaction for acid phosphatase was more intensive in glutaraldehyde fixation than in other fixatives. The lower concentration $(3.1 \%$ or $6.3 \%$ ) of glutaraldehyde seemed to be better than the high (12.5\%).

2) For preservation of the enzyme activity, 5 minutes were suitable for fixation of the smear materials, and 60 minutes were best for fixation of the coagulated materials.

3) For preservation of the structure of intracellular organelles of coagulated materials, the considerably long time was needed for fixation, but the longer 
time than 60 minutes disturbed very much the enzyme activity.

4) The incubation time in the substrate mixture needed 15 minutes on the smear materials and 30 minutes in the coagulated materials for complete demonstration of whole enzyme activity and for sufficient preservation of fine structure.

5) The histochemical reaction for acid phosphatase appeared clear cut in granular shape in the intracellular particular regions of the cytoplasm as shown in Fig. 1, and in the electron micrograph it was mainly demonstrated in lysosmes as shown in Fig. 2.

6) It was found that a non-specific precipitation for lead appeared sometimes in Golgi regions in AH-13 cancer cells, in spite of omitting the substrate from the medium.

\title{
Histochemical Distribution of Glutaminase Activated by Different Activators in the Rat Kidney
}

\author{
Yasuo Kishino
}

First Department of Surgery, Tokushima University School of Medicine, Tokushima

The most of ammonia in urine (60\%) is derived from glutamine by the action of glutaminase present in the kidney. The closed correlation between the magnitude of ammonia excretion and kidney glutaminase I activity has been already reported ${ }^{1}$, and Karnovsky et $\mathrm{al}^{2)}$ has made studies of the histochemical demonstration of glutaminase I activated by phosphate in the kidney of rat and hamster. Recently, Katunuma ${ }^{3)}$ suggested biochemically the presence of glutaminase activated by bicarbonate at $\mathrm{pH}$ 7.1. Since, up to the present time, little demonstration of histochemical localization of glutaminase activity with different activators (phosphate and bicarbonate existed as the two most important buffer system in the body fluid) has been obtained in any species. The author studied the histochemical distribution of the both glutaminases in the normal and pathologic kidney of the rat. On the other hand, the remainder of ammonia produced is derived from the $\alpha$-amino acids by the transaminationdeamination reaction ${ }^{4}$. Therefore, the author studied also the histochemical localization of transaminase $(\mathrm{GOT})^{\text {s) }}$ and glutamic dehydrogenase ${ }^{6)}$. In the acutely acidotic rats, the demonstration of carbonic anhydrase ${ }^{7)}$ was performed.

\section{Experimental Methods}

1) Preparation of tissues

The fresh kidney slices of rats were sectioned, $10-15 \mu$ in thickness, by 